\title{
Estudio cualitativo sobre la implementación del Programa de continuidad de cuidados y rehabilitación para personas con trastornos mentales graves en el Perú
}

\author{
Francisco Diez-Canseco, ${ }^{1}$ Jefferson Rojas-Vargas, ${ }^{1}$ Mauricio Toyama, ${ }^{1}$ María Mendoza, ${ }^{2}$ \\ Victoria Cavero, ${ }^{1}$ Humberto Maldonado, ${ }^{2}$ July Caballero ${ }^{2}$ y Yuri Cutipé ${ }^{2}$
}

Forma de citar Diez-Canseco F, Rojas-Vargas J, Toyama M, Mendoza M, Cavero V, Maldonado H, et al. Estudio cualitativo sobre la implementación del Programa de continuidad de cuidados y rehabilitación para personas con trastornos mentales graves en el Perú. Rev Panam Salud Publica. 2020;44:e134. https://doi.org/10.26633/RPSP.2020.134

RESUMEN Objetivo. Describir la implementación del Programa de continuidad de cuidados y rehabilitación (PCC-R) en centros de salud mental comunitaria (CSMC) del Perú.

Métodos. Estudio cualitativo sobre la implementación del PCC-R en cuatro CSMC de Lima y La Libertad, Perú. Se realizaron 42 entrevistas semiestructuradas individuales y un grupo focal con cinco participantes, para un total de 47 informantes entre usuarios, familiares y profesionales vinculados al diseño, la implementación y el monitoreo del PCC-R.

Resultados. El PCC-R es un programa central de los servicios de salud mental comunitaria en Perú, goza de amplia aceptación y los prestadores y usuarios refieren estar satisfechos con sus resultados. Sin embargo, el programa carece de un documento normativo que detalle sus objetivos, organización y actividades, lo que explicaría la variabilidad en su aplicación. Existen limitaciones en la gestión de recursos financieros y humanos del PCC-R y necesidades de capacitación y supervisión, que deben ser mejoradas. Existe consenso sobre la necesidad, la utilidad y la viabilidad de desarrollar un sistema de monitoreo del PCC-R. Se recogen buenas prácticas y sugerencias para enfrentar estos retos.

Conclusiones. El PCC-R es un programa insignia de los CSMC y de la reforma de la salud mental en Perú, y ha logrado amplia aceptación entre los prestadores y usuarios. Su implementación combina aciertos y dificultades, y revela tareas pendientes como desarrollar un documento normativo, mejorar la gestión de recursos, fortalecer la capacitación y acompañamiento, y aplicar un sistema de monitoreo para favorecer la mejora continua del programa.

Palabras clave Continuidad de la atención al paciente; salud mental; centros comunitarios de salud mental; investigación cualitativa; Perú.

Si bien los trastornos neuropsiquiátricos afectan a uno de cada cuatro peruanos $(1,2)$ y son la principal causa de carga de enfermedad en el país (12,7\%) (3), entre $75 \%$ y $85 \%$ de quienes requieren atención en salud mental no la reciben $(1,2)$. Para reducir esta brecha, el Perú inició una reforma de la atención en salud mental (4,5): en 2012 se aprobó la Ley 29889 que

\footnotetext{
CRONICAS Centro de Excelencia en Enfermedades Crónicas, Universidad Peruana Cayetano Heredia, Lima, Perú. $₫$ Francisco Diez-Canseco, fdiezcanseco@upch.pe
}

garantizaba la provisión de servicios de salud mental, y en 2014 se estableció un programa presupuestal para asegurar dichos servicios $(6,7)$. La reforma, encabezada por la Dirección de Salud Mental (DSAME) del Ministerio de Salud del Perú (MINSA), impulsa un modelo de atención comunitario (figura 1), que tiene entre sus pilares la creación de centros de

\footnotetext{
2 Dirección de Salud Mental, Ministerio de Salud del Perú.
} 
FIGURA 1. Modelo de atención comunitaria de salud del Perú y el PCC-R

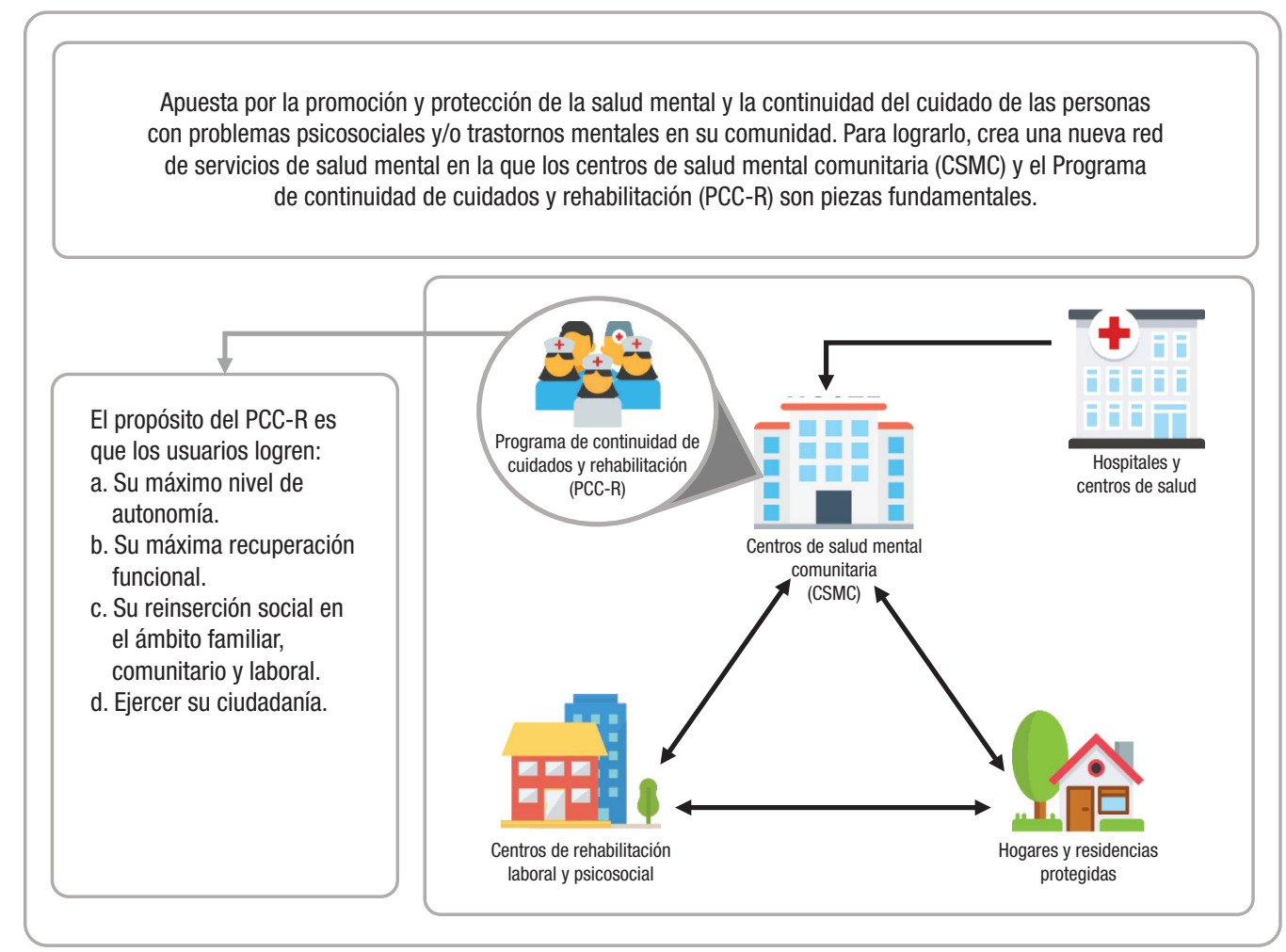

Fuente: elaboración propia a partir de las referencias 6 y 9.

salud mental comunitaria (CSMC) destinados a atender a personas con problemas de salud mental complejos. En la actualidad existen 155 CSMC y en 2021 deberían ser 281 en todo el Perú (8).

Un programa central en los CSMC es el Programa de continuidad de cuidados y rehabilitación (PCC-R), dirigido sobre todo a personas con trastornos mentales graves (TMG) cuya discapacidad y dependencia psicosocial requiere cuidados frecuentes y prolongados $(9,10)$. La evidencia internacional demuestra la efectividad de estos programas en personas con TMG, con mejora en su funcionamiento social, permanencia y satisfacción con el tratamiento, y disminución de la gravedad de los síntomas y el tiempo de hospitalización $(11,12)$.

En Perú, un gestor de caso monitorea a cada usuario del PCC-R, y se encarga de acompañarlo y garantizar su atención oportuna, dentro y fuera del CSMC, en el marco de un plan de continuidad de cuidados individualizado (PCCI) (9).

En los primeros años de la reforma, los esfuerzos se han enfocado en garantizar la creación y sostenibilidad de los CSMC, en los que se debía aplicar el PCC-R con base en lineamientos generales estipulados en el documento normativo de los CSMC (9). Considerado el tiempo transcurrido y la cantidad de CSMC, existe la necesidad de saber cómo ha sido el funcionamiento del PCC-R e identificar lecciones para mejorar su aplicación.

El presente estudio, realizado por la DSAME y CRONICAS Centro de Excelencia en Enfermedades Crónicas de la Universidad Peruana Cayetano Heredia (UPCH), se propuso describir la implementación del PCC-R, caracterizar su organización y principales actividades, evaluar su aceptabilidad y adopción, y recoger sugerencias para su mejora.

\section{MATERIALES Y MÉTODOS}

Para alcanzar nuestro objetivo, se realizó un estudio cualitativo, metodología que permite describir el estado actual del PCC-R desde las percepciones y experiencias de sus actores principales (13).

El estudio forma parte de la iniciativa Mejoras de la Ejecución de los Programas a través de la Investigación en Implementación (iPIER, del inglés Improving Program Implementation through Embedded Research), desarrollada por la Alianza para la Investigación en Políticas y Sistemas de Salud en colaboración con la Organización Panamericana de la Salud (OPS). Esta iniciativa coloca a los ejecutores de programas en el centro de una investigación, con el objetivo de entender las fallas en los sistemas de salud que crean barreras a la ejecución, lo que permite identificar soluciones y contribuye a la efectividad de los programas y políticas de salud (14).

El estudio se realizó en cuatro CSMC, dos de Lima, capital del Perú, y dos de La Libertad, en la costa norte, elegidos por ser los de mayor experiencia en la aplicación del PCC-R. En cada región, se planificó invitar a seis gestoras de caso (12 en total), dos estadísticos de los CSMC (cuatro en total), ocho usuarios (16 en total), cuatro familiares (ocho en total) y uno o dos profesionales locales de salud mental. A estos se sumarían seis funcionarios del nivel central del MINSA, cinco de la DSAME y uno de la Oficina General de Tecnologías de la Información (OGTI), con un total de 47 o 48 informantes.

Para seleccionar a los participantes se usó un muestreo por conveniencia (15). Los criterios de inclusión fueron ser adulto, dar consentimiento informado, tener un mínimo de seis 
meses en el PCC-R (usuarios y familiares) y tener la mayor antigüedad en el PCC-R de cada CSMC (gestoras).

Las técnicas cualitativas elegidas fueron el grupo focal para los decisores de políticas de la DSAME y la entrevista semiestructurada para los demás. Se diseñó una guía de grupo focal y seis guías de entrevista, una por perfil: gestoras, estadísticos, usuarios, familiares, funcionarios locales y funcionario de OGTI. Se utilizó una ficha para recolectar información sociodemográfica: sexo, edad, ocupación y tiempo en el PCC-R.

Antes de recolectar los datos, el estudio fue presentado en Lima y La Libertad. Los decisores de la DSAME colaboraron con la selección de funcionarios locales y gestoras y, estas últimas, con la selección de usuarios y familiares. Tras elegirlos, se verificaron los criterios de inclusión y se obtuvo el consentimiento informado. Se grabaron las entrevistas y el grupo focal, previo consentimiento de los participantes, las cuales fueron realizadas y analizadas por el equipo de CRONICAS.

Se transcribieron y codificaron los audios mediante el software ATLAS.ti 7.5. Se realizó un análisis de contenido, cuyo propósito es organizar y categorizar los datos recogidos para interpretarlos y extraer conclusiones sustanciales (16). La adopción y aceptabilidad del PCC-R fueron definidas, respectivamente, como: i) la intención, decisión inicial o acción para poner en práctica las actividades del programa, y ii) la percepción de los actores involucrados de que el programa es agradable o satisfactorio (17). Para cada perfil de informante, se creó un libro de códigos con categorías descriptivas para organizar los hallazgos. Los códigos iniciales se diseñaron con base en los temas de las guías de entrevista y se sumaron temas nuevos surgidos en la codificación. Se estandarizó la codificación entre los investigadores y se triangularon las miradas de distintos perfiles.

El estudio fue aprobado por el Comité Institucional de Ética de la UPCH y el Comité de Revisión Ética de la OPS (PAHOERC). Todos los informantes participaron de manera voluntaria tras firmar un consentimiento informado y, para proteger su identidad, sus nombres se reemplazaron por códigos y se omitió información que facilitara su identificación.

\section{RESULTADOS}

Se realizaron 42 entrevistas y un grupo focal, con un total de 47 participantes (cuadro 1).
Los decisores y las gestoras refirieron que el PCC-R combina elementos de distintos modelos, como el "manejo de casos" (18) y el "tratamiento asertivo comunitario" (19), e incluye herramientas adaptadas de otros programas de salud locales.

Aunque se aplica desde 2016, el PCC-R no cuenta con un documento normativo específico que describa en detalle sus objetivos, organización, actividades y monitoreo. Existe, más bien, una propuesta con lineamientos generales compartida con los CSMC nuevos, que fueron adaptando a su contexto y recursos. Así, los cuatro CSMC ya aplicaban el PCC-R, con una visión general común, pero con importantes diferencias. Algunas coincidencias identificadas eran: i) el PCC-R está dirigido a personas con TMG, ii) ofrece cuidado integral, constante y cercano, iii) busca recuperar habilidades perdidas, y iv) se articula con servicios intramuros y extramuros del CSMC. Los decisores y las gestoras destacaron la necesidad de contar con documentos técnicos del PCC-R que guíen la labor del personal de salud, y sean a la vez flexibles y consideren la diversidad de contextos socioculturales y de recursos.

Los CSMC cuentan con un equipo interdisciplinario de profesionales vinculado al PCC-R (psiquiatra, psicólogo y terapista ocupacional, entre otros), aunque suelen ser las enfermeras quienes asumen el rol de gestoras y coordinadoras. La coordinadora organiza y supervisa el funcionamiento del PCC-R. Cada gestora tiene asignados entre tres y seis usuarios, con quienes debe diseñar un PCCI adaptado a sus necesidades. Junto a los otros profesionales del CSMC debe realizar, coordinar y monitorear el cumplimiento de las actividades del PCCI.

Las actividades del PCC-R son similares en todos los CSMC; varían en cantidad y frecuencia, según la gravedad del cuadro y las necesidades de cada usuario (cuadro 2). Todos los CSMC clasifican la gravedad mediante un "semáforo": rojo (alta), amarillo (media) y verde (baja).

La capacitación del personal de salud vinculado al PCC-R ha sido muy variable, en metodología, calidad y duración. Algunos profesionales realizaron pasantías de hasta seis meses en el extranjero, donde observaron cómo se ejecutaba el PCC-R y realizaron cursos virtuales. La mayoría de las gestoras recibieron entrenamiento bajo el modelo de cascada (20), en réplicas de uno a tres días realizadas por las coordinadoras, con revisión de los materiales de capacitaciones del MINSA. La mayoría de las gestoras recibió una sola sesión de entrenamiento: las sesiones de refuerzo o actualización no eran habituales. Para los decisores,

CUADRO 1. Participantes por región, tipo de informante, duración y técnica utilizada

\begin{tabular}{|c|c|c|c|c|c|c|c|c|c|}
\hline \multirow{2}{*}{$\begin{array}{c}\text { Técnica de recolección } \\
\text { de datos }\end{array}$} & \multirow[t]{2}{*}{ Informantes } & \multirow{2}{*}{$\begin{array}{l}\text { Duración } \\
\text { (minutos) }\end{array}$} & \multicolumn{2}{|c|}{ Nacional } & \multicolumn{2}{|c|}{ Lima } & \multicolumn{2}{|c|}{ La Libertad } & \multirow[t]{2}{*}{ Total } \\
\hline & & & $\mathrm{H}$ & $\mathrm{M}$ & $\mathrm{H}$ & $\bar{M}$ & $\mathrm{H}$ & $\mathrm{M}$ & \\
\hline Grupo focal & Decisores de políticas de la DSAME & 199 & 1 & 4 & & & & & 5 \\
\hline \multirow[t]{6}{*}{ Entrevistas semiestructuradas } & Funcionario de la OGTI & 62 & 1 & & & & & & 1 \\
\hline & Funcionarios locales de salud mental & 101 & & & & & & 1 & 1 \\
\hline & Estadísticos locales & $43-100$ & & & 3 & & & 2 & 5 \\
\hline & Gestoras de caso del PCC-R & $63-147$ & & & & $6^{\mathrm{a}}$ & $1^{b}$ & $5^{c}$ & 12 \\
\hline & Usuarios del PCC-R & $21-58$ & & & 3 & 4 & 4 & 4 & 15 \\
\hline & Familiares de los usuarios & $26-48$ & & & 1 & 3 & & 4 & 8 \\
\hline Total & & & 2 & 4 & 7 & 13 & 5 & 16 & 47 \\
\hline
\end{tabular}

DSAME, Dirección de Salud Mental; OGTI, Oficina General de Tecnologías de la Información; PCC-R, Programa de continuidad de cuidados y rehabilitación; H, hombres; M, mujeres.

aSeis enfermeras.
bUn médico de familia.

bUn médico de familia.
'Cuatro enfermeras y una rehabilitadora física.

Fuente: elaboración propia a partir de resultados. 


\section{CUADRO 2. Tipo y frecuencia de actividades del PCC-R según el nivel de gravedad del cuadro del usuario}

\begin{tabular}{|c|c|c|c|}
\hline \multirow[t]{2}{*}{ Actividades } & \multicolumn{3}{|c|}{ Nivel de gravedad } \\
\hline & Alto (rojo) & Medio (amarillo) & Bajo (verde) \\
\hline Visitas domiciliarias para evaluación, intervención y seguimiento & Diario 0 interdiario & Semanal & Mensual \\
\hline Llamadas telefónicas como parte del seguimiento & Interdiario & Semanal & Mensual \\
\hline Consultas o citas para psiquiatría, psicología y terapia ocupacional & Semanal & $2-3$ veces al mes & Mensual \\
\hline Psicoeducación a usuarios y familiares sobre el trastorno mental y la medicación & 4 veces en 3 meses o menos & & \\
\hline $\begin{array}{l}\text { Acompañamiento o coordinación para trámites: afiliación a un seguro y obtener el } \\
\text { documento de identidad, entre otros }\end{array}$ & 1-3 veces en 1 semana & & \\
\hline Visitas a espacios recreativos que promuevan la autonomía & & & Semestral \\
\hline
\end{tabular}

Fuente: elaboración propia a partir de resultados.

la variabilidad en el entrenamiento explicaría, en parte, las diferencias de aplicación del PCC-R en los diferentes CSMC.

La opinión de las gestoras sobre la capacitación fue, en general, positiva, aunque la mayoría manifestó la necesidad de un entrenamiento más intensivo y práctico, brindado por profesionales que hayan aplicado el PCC-R.

Además, profesionales de Lima señalaron que no recibían acompañamiento en el programa, mientras en La Libertad indicaron que la supervisión que recibían era general, sobre su labor en el CSMC y que, en relación con el PCC-R, se limitaba a verificar la documentación. Los decisores añadieron que el PCC-R no contaba con un documento normativo para dichas actividades.

Si bien no existe un procedimiento estándar de inclusión de usuarios en el PCC-R, los informantes coinciden en que está dirigido a personas con TMG y detallan procesos de ingreso similares. Los candidatos al PCC-R se identifican durante la "acogida" al CSMC -evaluación de ingreso- o cuando un tratamiento no da resultado. La decisión de ingreso y la asignación del nivel de gravedad (rojo, amarillo o verde) suelen realizarse con base en valoraciones clínicas y psicosociales que los profesionales comparten en sus reuniones, el uso de escalas es poco frecuente. Tras decidirse el ingreso del usuario, la coordinadora del PCC-R lo asigna a una gestora; para esto, tiene en cuenta tanto la carga de trabajo de las gestoras como la gravedad del cuadro del usuario.

La elaboración del PCCI mediante un formato estandarizado es una tarea común en los CSMC. El plan detalla los objetivos del usuario en el PCC-R, las actividades para lograrlos, los profesionales responsables y los plazos.

Si bien es una de sus premisas, el PCCI suele diseñarse sin la participación de los usuarios, en parte porque al ingresar al PCC-R suelen tener dificultades para expresar su consentimiento. Son los familiares quienes reciben la explicación sobre el programa y firman el consentimiento, aunque sin participar de manera activa en la construcción del PCCI.

Los usuarios entrevistados tenían una permanencia mayor a seis meses en el PCC-R, y aunque no todos lo reconocen por su nombre, sí identifican sus actividades y señalan que estas "apoyan la salud mental", "cuidan" y "mejoran". Los familiares sí reconocen el programa, y señalan que se "acompaña" de manera constante al usuario para su "rehabilitación" y "mejora".

El tiempo de permanencia de los usuarios en el PCC-R es variable tanto dentro como entre los CSMC. Una razón es que existen diferencias entre los usuarios -por ejemplo, en su necesidad de soporte o sus recursos- y las metas que se establecen para cada uno. Otra razón es que los CSMC parecen tener miradas distintas sobre la finalidad del PCC-R, que se expresan en la permanencia de los usuarios. Así, en un CSMC de Lima la estadía máxima prestablecida era de seis meses, lo que se refleja en un PCCI con objetivos más acotados, mientras en los otros CSMC la estancia era más prolongada, con pocas altas médicas. Esta situación difiere de lo que algunas gestoras aprendieron durante su capacitación:

"La teoría te dice que por cada color es aproximadamente un trimestre: un trimestre en rojo, un trimestre en amarillo, un trimestre en verde (...) O sea, en teoría son nueve meses en el PCC, ¿no? En la vida real no... puede estar un mes, dos meses, un año, dos años. Uno está desde el 2016 hasta ahora, pero por la complejidad de su caso" (gestora de Lima).

\section{Adopción y aceptabilidad del PCC-R}

La adopción del PCC-R se manifestó en la intención, habitual entre las gestoras, de aplicar y efectivizar las distintas actividades del PCC-R. Aunque existe variabilidad entre los CSMC, se ha identificado un conjunto importante de actividades comunes, como la psicoeducación a usuarios y familiares y la intervención para mejorar las actividades de la vida diaria y las actividades recreativas, realizadas a través de métodos habituales como visitas domiciliarias, llamadas y reuniones de seguimiento del equipo del CSMC.

Los informantes, tanto a nivel local como a nivel central, identificaron barreras para adoptar las actividades del PCC-R, en su mayoría relacionadas con la gestión de recursos económicos y humanos, y de apoyo al programa. Asimismo, ofrecieron posibles soluciones (cuadro 3 ).

Las gestoras manifiestan una gran aceptabilidad del PCC-R, lo que se percibe también por los decisores. Las gestoras indican que es un programa "necesario" e "importante", que permite alcanzar buenos resultados trabajando en equipo. Como aspectos positivos, se destacan el mayor acompañamiento a los usuarios, y poder conocerlos mejor. Por último, con respecto a los resultados del programa, todas las gestoras estaban satisfechas, aunque había también propuestas para mejorarlo, como estandarizar su funcionamiento, incrementar la capacitación y aumentar el compromiso del equipo del CSMC para cumplir las actividades.

"Este programa para mí es muy importante, muy valioso, me siento mejor trabajando en equipo porque trabajar de a uno es imposible" (gestora de Lima). 
CUADRO 3. Dificultades y sugerencias para la adopción del PCC-R

\begin{tabular}{|c|c|c|}
\hline Ámbito & Dificultades & Sugerencias \\
\hline $\begin{array}{l}\text { Gestión de recursos } \\
\text { económicos }\end{array}$ & $\begin{array}{l}\text { Falta de dinero para visitas domiciliarias y llamadas a usuarios } \\
\text { Procedimientos complicados y lentos para solicitar y recibir fondos }\end{array}$ & $\begin{array}{l}\text { Aumentar presupuesto del PCC-R } \\
\text { Incluir el transporte para actividades extramurales en la norma técnica del PCC-R } \\
\text { Hacer alianzas con instituciones locales que brinden apoyo (p. ej., transporte) }\end{array}$ \\
\hline Apoyo al PCC-R & $\begin{array}{l}\text { Priorización de la cantidad sobre la calidad de actividades } \\
\text { entre directivos del sistema de salud: "el PCC-R realiza menos } \\
\text { actividades que otros servicios" } \\
\text { Rechazo al modelo comunitario entre algunos jefes de CSMC, } \\
\text { lo que impide actividades del PCC-R, como salidas a campo } \\
\text { Apoyo limitado de profesionales del CSMC a actividades del } \\
\text { PCC-R, ya que se considera que es responsabilidad de enfermeras }\end{array}$ & $\begin{array}{l}\text { Sensibilizar a directivos y equipos de CSMC sobre el modelo comunitario de } \\
\text { salud mental } \\
\text { Incluir como gestores a otros profesionales del CSMC para comprometerlos con } \\
\text { el PCC-R } \\
\text { Incluir el desempeño del PCC-R como un indicador clave para que sea prioridad }\end{array}$ \\
\hline
\end{tabular}

PPC-R, Programa de continuidad de cuidados y rehabilitación; CSMC, Centro de salud mental comunitaria.

Fuente: elaboración propia a partir de resultados.

Los usuarios y los familiares también manifestaron una gran aceptación del PCC-R. La mayoría estaba "cómodo" o "satisfecho" con la atención y el trato amable del personal, y destacaron su "interés", "comprensión" y "escucha". Los usuarios dijeron estar contentos, pues percibían mejoras a nivel físico, emocional y social, reflejadas en la disminución de síntomas y en la mayor integración en espacios comunitarios y laborales.

\section{Monitoreo del PCC-R: situación actual y propuestas}

El PCC-R no cuenta aún con procedimientos, instrumentos e indicadores de monitoreo. Los CSMC suelen registrar sus actividades en la historia clínica física y en el sistema de información HIS-MINSA. Este sistema tiene la limitación de registrar solo actividades (no registra resultados) y ofrecer información general del CSMC; no permite filtrar información del PCC-R, lo que impide su monitoreo adecuado.

Las gestoras señalaron que es inusual que sus superiores en la Región soliciten reportes de las actividades del PCC-R, aunque en ocasiones les piden datos específicos, como la cantidad de usuarios o visitas domiciliarias.

Las gestoras utilizan las historias clínicas y sus propios registros para dar seguimiento a los usuarios del PCC-R y monitorear el cumplimiento del PCCI. De esta manera, evalúan y reformulan las actividades.

Existe consenso sobre la necesidad, utilidad y viabilidad de tener un sistema de monitoreo del PCC-R, lo que permitiría reflejar mejor las actividades de las gestoras y mejorar el seguimiento de los usuarios.

Asimismo, hay acuerdo en utilizar el sistema HIS-MINSA para el registro de varios de los datos necesarios para monitorear el PCC-R, porque es un sistema "confiable", "conocido", y "fácil de usar". Los decisores indicaron que, para lograrlo, deberían introducirse algunos cambios en el HIS-MINSA (p. ej., permitir filtrar a los usuarios del PCC-R) y estandarizar los códigos para reportar las actividades del programa.

Varios decisores del nivel central mostraron disposición para aplicar, además, un sistema de registro complementario del PCC-R, que permitiera, en plazos cortos, recabar datos clave para el monitoreo. La barrera mencionada con mayor frecuencia para este registro nuevo fue la carga de trabajo que supondría para estadísticos y gestoras.
Los informantes del sistema de salud sugirieron indicadores clave para evaluar el PCC-R (cuadro 4), que se agrupan en indicadores descriptivos y de proceso, sobre los usuarios y actividades del PCC-R; e indicadores de calidad y resultados del PCC-R.

Por su parte, los familiares y los usuarios ofrecieron recomendaciones para mejorar la evaluación que se les hace en el PCC-R, que deberían considerarse también en el diseño de los indicadores (cuadro 4).

\section{DISCUSIÓN}

Nuestro estudio describe la implementación del PCC-R desde la mirada de sus protagonistas, pone en evidencia aciertos, dificultades, similitudes y diferencias en la marcha del programa, y recoge sugerencias para mejorarlo.

El PCC-R se viene consolidando como una estrategia central de los servicios de salud mental comunitaria en Perú, tiene amplia aceptación por los actores involucrados y existe satisfacción general con sus resultados. Sin embargo, existen también tareas pendientes que mejorarían su implementación, que se hacen más necesarias si se tiene en cuenta que el PCC-R funcionará en casi 300 CSMC en los próximos años.

A continuación, se discuten los hallazgos y se ofrecen recomendaciones sobre cuatro dimensiones esenciales del PCC-R: la normatividad, la ejecución de actividades, la capacitación y el acompañamiento, y el monitoreo y la evaluación.

\section{Normatividad}

Existe la necesidad de desarrollar un documento que describa los aspectos esenciales y comunes del PCC-R -desde sus objetivos hasta su monitoreo-, lo que facilitaría su implementación funcionamiento y evaluación. La aplicación exitosa de estos documentos normativos permite mejorar la calidad de los procedimientos y la atención a los usuarios $(21,22)$. A la vez, es importante garantizar que el PCC-R pueda adaptarse a distintas realidades y usuarios con diversas condiciones de salud, necesidades y recursos $(23,24)$. La norma técnica del PCC-R está en elaboración y se nutrirá de los resultados de esta investigación. 
CUADRO 4. Propuestas de indicadores y temas a explorar en la evaluación de usuarios del PCC-R

\begin{tabular}{|c|c|c|}
\hline \multicolumn{2}{|c|}{ Informante } & Descripción \\
\hline $\begin{array}{l}\text { Sugerencias de profesionales } \\
\text { de salud y decisores de } \\
\text { políticas locales sobre } \\
\text { indicadores }\end{array}$ & Descriptivos y de proceso & $\begin{array}{l}\text { Descripción de usuarios del PCC-R } \\
\text { Número de usuarios del PCC-R, porcentaje de usuarios con TMG, porcentaje de usuarios según la gravedad } \\
\text { del cuadro, porcentaje de usuarios certificados con discapacidad, tiempo de permanencia en el PCC-R, } \\
\text { porcentaje de usuarios que adhieren al tratamiento, porcentaje de usuarios que abandonan el PCC-R. } \\
\text { Actividades del PCC-R } \\
\text { Número de reuniones del equipo del PCC-R, número de visitas domiciliarias a usuarios, número de } \\
\text { llamadas a usuarios, número de PCCI cumplidos en su totalidad. }\end{array}$ \\
\hline \multicolumn{2}{|c|}{$\begin{array}{l}\text { Sugerencias de usuarios y familiares sobre temas a explorar en } \\
\text { evaluaciones del usuario }\end{array}$} & $\begin{array}{l}\text { Cambios en los síntomas y el desempeño social (en la familia y en el trabajo) } \\
\text { Dificultades para comprender, adaptarse y adherirse al tratamiento } \\
\text { Dificultades en retomar las actividades diarias }\end{array}$ \\
\hline
\end{tabular}

PCC-R, Programa de continuidad de cuidados y rehabilitación; TMG, trastornos mentales graves; PCCI, plan de continuidad de cuidados individualizado.

Fuente: elaboración propia a partir de resultados.

\section{Aplicación de actividades}

Aunque el PCC-R tiene alta aceptabilidad, su adopción enfrenta diversas limitaciones, desde una cultura organizacional que prioriza la cantidad sobre la calidad hasta la sobrecarga de las gestoras.

En primer lugar, se recomienda simplificar la solicitud, entrega y rendición de dinero, y establecer colaboraciones con instituciones locales que apoyen las actividades del PCC-R. La evidencia indica que es esencial garantizar la disponibilidad de recursos para mejorar el funcionamiento y sostenibilidad de un programa $(25,26)$. Segundo, se deben establecer parámetros para evaluar la productividad del PCC-R, un programa que apuesta por un acompañamiento intensivo y prolongado de pocos usuarios y, por lo tanto, no se puede medir con los mismos criterios que otros. Tercero, es necesaria la asignación de personal a dedicación exclusiva al PCC-R, para mejorar su gestión y garantizar el cumplimiento de sus actividades y monitoreo. Cuarto, se debe fomentar la participación de usuarios desde su ingreso al PCC-R. Por último, se sugiere identificar y compartir buenas prácticas de los CSMC.

\section{Capacitación y acompañamiento}

Los aspectos clave para la implementación exitosa de un programa, sobre todo uno reciente, son la capacitación continua y el acompañamiento del personal (25). Se recomienda incorporar capacitaciones intensivas que garanticen contenidos esenciales a todo el personal del programa. A su vez, es necesario generar espacios regulares de acompañamiento e interaprendizaje entre gestoras y con expertos externos, para aclarar dudas, recibir retroalimentación y elaborar soluciones en conjunto. Como lo muestra la literatura, las herramientas tecnológicas son útiles para facilitar el acceso al acompañamiento y la capacitación $(27,28)$.

\section{Monitoreo y evaluación}

El riguroso monitoreo y evaluación es un elemento esencial no solo para la sostenibilidad (26), sino también para la mejora
(25) de los programas de salud. La norma futura del PCC-R debe incluir un sistema de monitoreo con indicadores de proceso, calidad y resultado, que incluya indicadores relevantes para usuarios y familiares (29), y un registro sencillo desde el punto de vista tecnológico y poco demandante para el personal, con el fin de garantizar su utilización. Estos elementos son clave en programas de salud efectivos, puesto que permiten obtener información precisa y oportuna sobre su implementación y su impacto a largo plazo (25).

El sistema debería poder implementarse en un tiempo corto y permitir un seguimiento adecuado del PCC-R a nivel central, regional y local. Para lograrlo, se recomienda mejorar el sistema HIS-MINSA actual y complementarlo con un registro simple que asegure la información para estimar los indicadores. Estas fuentes de información serán útiles para evaluar el estado del programa, con el fin de brindar retroalimentación periódica que permita su evaluación y mejora (25). Además, se recomienda realizar evaluaciones externas para conocer la satisfacción y las sugerencias de usuarios y familiares, algo que ha demostrado mejorar la calidad de la atención, así como los resultados del tratamiento recibido, al centrarse en sus necesidades $(30,31)$.

Por último, se deberían realizar estudios piloto del sistema nuevo, para refinar los procedimientos e indicadores, antes de escalarlo a todos los CSMC del país.

Por el tiempo y los recursos disponibles, el trabajo de campo se concentró en cuatro de los CSMC más antiguos del Perú, lo que no representa necesariamente la realidad de otros CSMC más nuevos. Aun así, dado que los primeros han sido un modelo, y en muchos casos responsables del entrenamiento de los segundos, se esperaría que existan varios procedimientos y retos similares.

Por otro lado, las entrevistas con los usuarios del PCC-R, algunas veces por su situación de salud y otras veces quizás por deseabilidad no siempre daban lugar a mucha información, en particular a críticas o sugerencias sobre el programa. Más allá de la necesidad que existe de seguir trabajando en metodologías que permitan garantizar la expresión de los usuarios de servicios de salud mental, la inclusión de los familiares permitió, en cierta medida, recoger la mirada de quienes reciben los servicios del PCC-R. 


\section{CONCLUSIONES}

El PCC-R es un programa insignia de los CSMC y de la reforma de la atención de salud mental en Perú, que ha logrado gran aceptabilidad entre los prestadores y los usuarios. Su implementación combina aciertos y dificultades, y revela tareas pendientes como desarrollar un documento normativo, mejorar la gestión de recursos, fortalecer la capacitación y acompañamiento, e implementar un sistema de monitoreo para favorecer la toma de decisiones informadas y la mejora continua del programa.

Contribución de los autores. YC/FDC/MM/MT/VC/HC/ JC contribuyeron a la conceptualización del estudio y adquisición de fondos. YC/FDC/MM se encargaron de la supervisión de actividades. FDC/MT/VC/JRV realizaron la recopilación y análisis de datos, preparación de borradores, revisión de documentos y edición. Todos los autores revisaron y aprobaron el manuscrito final.
Agradecimientos. Los autores agradecen a los funcionarios de nivel central del MINSA, funcionarios locales de salud mental, profesionales de los CSMC, usuarios y familiares por la información que han brindado. Los autores también agradecen a Jimena Rivas, Ana Lucía Vilela y Jill Portocarrero por su apoyo en la transcripción de audios y codificación de entrevistas.

\section{Conflicto de intereses. Ninguno declarado por los autores.}

Apoyo financiero. Esta investigación fue posible gracias al financiamiento de la Organización Panamericana de la Salud en la convocatoria "Incorporación de la investigación para avanzar en el cumplimiento de los Objetivos de Desarrollo Sostenible (ER-SDG)".

Declaración. Las opiniones expresadas en este manuscrito son responsabilidad del autor y no reflejan necesariamente los criterios ni la política de la RPSP/PAJPH y/o de la OPS.

\section{REFERENCIAS}

1. Instituto Nacional de Salud Mental "Honorio Delgado Hideyo Noguchi". Estudio epidemiológico de salud mental en Lima Metropolitana y Callao - Replicación 2012. Anales de Salud Mental. 2013;29. Disponible en: http://www.insm.gob.pe/investigacion/ archivos/estudios/2012 ASM -EESM -LM.pdf

2. World Health Organization. Mental Health Atlas 2011. Country profile: Peru. Geneve: WHO; 2011. Disponible en: https://www. who.int/mental_health/publications/mental_health_atlas_2011/ en /\#: : text=Overview, $98 \% 25 \% 20$ of $\% 20$ the $\% 20$ world's $\% 20$ population.

3. Ministerio deSalud delPerú(MINSA). Carga deenfermedad enel Perú: estimación delos años de vida saludables perdidos 2016. Lima:MINSA; 2018. Disponible en: https://www.gob.pe/institucion/minsa/ informes-publicaciones / 276778-carga-de-enfermedad-en-el-peruestimacion-de-los-anos-de-vida-saludables-perdidos-2016

4. Toyama M, Castillo H, Galea JT, Brandt LR, Mendoza M, Herrera V, et al. Peruvian mental health reform: a framework for scaling-up mental health sServices. Int J Heal Policy Manag. 2017;6(9):501-8.

5. Miranda JJ, Diez-Canseco F, Araya R, Cutipe Y, Castillo H, Herrera $\mathrm{V}$, et al. Transitioning mental health into primary care. The Lancet Psychiatry. 2017;4(2):90-2.

6. Gobierno del Perú. Reglamento de la Ley N. ${ }^{\circ}$ 29.889. Decreto Supremo $\mathrm{N}^{\circ}$ 033-2015-SA. Disponible en: http:/ / www.aprodeh.org. pe/documentos/marco-normativo/discapacidad/Reglamento_ de_la_Ley_N_29889_Ley_que_modifica_el_articulo_11_de_la_ Ley_26842_Ley_General_de_Salud_y_garantiza_los_derechos_de _ las_personas_con_problemas_de_salud_mental.pdf

7. Ministerio de Economía y Finanzas del Perú. Programa presupuestal 0131: control y prevención en salud mental. Disponible en: https:// www.mef.gob.pe/contenidos/presu_publ/ppr/prog_presupuestal/ articulados/0131_control_prevencion_salud_mental.pdf

8. Ministerio de Salud del Perú (MINSA). Plan nacional de fortalecimiento de servicios de salud mental comunitaria 2018-2021. Lima: MINSA; 2018.

9. Ministerio de Salud del Perú (MINSA). Norma técnica de salud: centros de salud mental comunitarios. Lima: MINSA; 2017.

10. Cubillos L, Muñoz J, Caballero J, Mendoza M, Pulido A, Carpio K, et al. Addressing severe mental illness rehabilitation in Colombia, Costa Rica, and Peru. Psychiatr Serv. 2020;71(4):378-84.

11. Dieterich M, Irving CB, Bergman H, Khokhar MA, Park B, Marshall $\mathrm{M}$. Intensive case management for severe mental illness. Cochrane Database Syst Rev. 2017;(1).

12. Adair CE, McDougall GM, Mitton CR, Joyce AS, Wild TC, Gordon $\mathrm{A}$, et al. Continuity of care and health outcomes among persons with severe mental illness. Psychiatr Serv.2005;56(9):1061-9.
13. Pedraz A, Zarco J, Ramasco M, Palmar AM. Investigación cualitativa. $1^{\circ}$ ed. Barcelona: Elsevier; 2014.

14. Langlois E V, Mancuso A, Elias V, Reveiz L. Embedding implementation research to enhance health policy and systems: a multi-country analysis from ten settings in Latin America and the Caribbean. Heal Res Policy Syst. 2019;17(1):85.

15. Teddlie C, Yu F. Mixed methods sampling. J Mix Methods Res. 2007;1(1):77-100.

16. Bengtsson M. How to plan and perform a qualitative study using content analysis. NursingPlus Open. 2016;2:8-14.

17. Proctor E, Silmere H, Raghavan R, Hovmand P, Aarons G, Bunger A, et al. Outcomes for implementation research: conceptual distinctions, measurement challenges, and research agenda. Adm Policy Ment Heal. 2011;38(2):65-76.

18. Bachrach LL. Continuity of care and approaches to case management for long-term mentally ill patients. Psychiatr Serv. 1993;44(5): 465-8.

19. Bond GR, Drake RE, Mueser KT, Latimer E. Assertive community treatment for people with severe mental illness. Dis Manag Heal Outcomes. 2001;9(3):141-59.

20. Davini M, Nervi L, Roschke M. La capacitación del personal de los servicios de salud en proyectos relacionados con los procesos de reforma sectorial. Lima: Organización Panamericana de la Salud; 2002: 91. Disponible en: http://bvs.minsa.gob.pe/local/minsa/2780.pdf

21. Heymann T. Clinical protocols are key to quality health care delivery. Int J Health Care Qual Assur.1994;7(7):14-7.

22. Graham ID, Harrison MB. Evaluation and adaptation of clinical practice guidelines. Evid Based Nurs. 2005;8(3):68-72.

23. May CR, Johnson M, Finch T. Implementation, context and complexity. Implement Sci. 2016;11(1):141te.

24. Squires JE, Graham ID, Hutchinson AM, Michie S, Francis JJ, Sales A, et al. Identifying the domains of context important to implementation science: a study protocol. Implement Sci. 2015;10 (1):135.

25. Frieden TR. Six components necessary for effective public health program implementation. Am J Public Health.2014;104(1):17-22.

26. Schell SF, Luke DA, Schooley MW, Elliott MB, Herbers SH, Mueller NB, et al. Public health program capacity for sustainability: a new framework. Implement Sci. 2013;8(1):15.

27. Evans S, Sonderlund A, Tooley G. Effectiveness of online interprofessional education in improving students' attitudes and knowledge associated with interprofessional practice. Focus Heal Prof Educ. 2013;14(2):12-20.

28. Islam MM, Parkinson A, Burns K, Woods M, Yen L. A training program for primary health care nurses on timely diagnosis and 
management of dementia in general practice: an evaluation study. Int J Nurs Stud. 2020;105:103550.

29. Kötter T, Schaefer F, Scherer M, Blozik E. Involving patients in quality indicator development: a systematic review. Patient Prefer Adherence. 2013;259-68.

30. Izadi A, Jahani Y, Rafiei S, Masoud A, Vali L. Evaluating health service quality: using importance performance analysis. Int J Health Care Qual Assur. 2017;30(7):656-63.

31. Reese RJ, Duncan BL, Kodet J, et al. Patient feedback as a quality improvement strategy in an acute care, inpatient unit: an investigation of outcome and readmission rates. Psychol Serv. 2018;15(4):470-6. Doi:10.1037/ser0000163

Manuscrito recibido el 30 de junio de 2020. Aceptado para su publicación, tras revisión, el 14 de setiembre de 2020.

\section{Qualitative study of the implementation of the Continuity of Care and Rehabilitation Program for people with severe mental disorders in Peru}

SUMMARY Objective. Describe the implementation of the Continuity of Care and Rehabilitation Program (PCC-R) in community mental health centers (CSMCs, Spanish acronym) in Peru.

Methods. Qualitative study of the implementation of the PCC-R in four CSMCs in Lima and La Libertad, Peru. Forty-two individual semi-structured interviews were conducted, as well as a focus group with five participants, for a total of 47 informants, including users, family members, and professionals involved in the design, implementation, and monitoring of the program.

Results. The PCC-R is a key program for community mental health services in Peru and it enjoys broad acceptability. Providers and users report satisfaction with its results; however, the program lacks a policy document specifying its objectives, organization, and activities. This would explain the variability in its implementation. The PCC-R has limitations in terms of management of financial and human resources, and it is necessary to improve training and supervision. There is consensus on the need, usefulness, and viability of developing a system to monitor the PCC-R. Good practices and suggestions are presented with a view to addressing these challenges.

Conclusions. The PCC-R is a flagship program for the CSMCs and for the mental health reform in Peru. It has achieved broad acceptability among providers and users. Its implementation combines successes and difficulties, with pending tasks that include developing a policy document, improving resource management, strengthening training and supervision, and implementing a monitoring system for continuous improvement of the program.

Keywords $\quad$ Continuity of patient care; mental health; community mental health centers; qualitative research; Peru. 


\section{Estudo qualitativo sobre a implementação do Programa de Continuidade de Cuidados e Rehabilitação para pessoas com transtornos mentais graves no Peru}

RESUMO Objetivo. Descrever a implementação do Programa de Continuidade de Cuidados e Reabilitação (PCC-R) em centros de saúde mental comunitária (CSMCs) do Peru.

Métodos. Estudo qualitativo sobre a implementação do PCC-R em quatro CSMCs em Lima e La Libertad, no Peru. Realizamos 42 entrevistas semiestruturadas individuais e um grupo focal com cinco participantes, com um total de 47 participantes, entre usuários, familiares e profissionais envolvidos na elaboração, implementação e monitoramento do PCC-R.

Resultados. O PCC-R é um programa central dos serviços de saúde mental comunitária no Peru, conta com ampla aceitabilidade e os prestadores e usuários dizem estar satisfeitos com seus resultados. No entanto, o programa não possui um documento normativo que detalhe os seus objetivos, organização e atividades, o que pode explicar a variabilidade em sua aplicação. Existem limitações na gestão dos recursos financeiros e humanos do PCC-R e necessidades de capacitação e supervisão, que devem ser melhoradas. Há consenso sobre a necessidade, utilidade e viabilidade de se desenvolver um sistema de monitoramento do PCC-R. Apresentamos aqui um conjunto de boas práticas e sugestões para enfrentar esses desafios.

Conclusões. O PCC-R é um programa emblemático dos CSMCs e da reforma da saúde mental no Peru, tendo obtido ampla aceitação entre prestadores e usuários. A sua implementação combina acertos e dificuldades e revela tarefas pendentes, tais como o desenvolvimento de um documento normativo, uma melhor gestão de recursos, o reforço da capacitação e supervisão e a aplicação de um sistema de monitoramento para fomentar a melhoria contínua do programa.

Palavras-chave Continuidade da assistência ao paciente; saúde mental; centros comunitários de saúde mental; pesquisa qualitativa; Peru. 